\title{
OVERBUILDING AND DEVELOPMENT CASCADES UNDER IRRATIONAL EXPECTATIONS
}

\author{
Xiu-Mei LV a, Gang-Zhi FAN b,* \\ a School of Finance, Chongqing Technology and Business University, 19 Xuefu Ave., Nan'an District, \\ Chongqing 400067, China \\ ${ }^{b}$ Department of Real Estate Studies, Konkuk University, 120 Neungdong-ro, Gwangin-gu, \\ Seoul 143-701, Korea
}

Received 24 November 2013; accepted 10 July 2014

\begin{abstract}
The recent sluggish recovery in the U.S. house market has further motivated our research interests in overbuilding in real estate markets. Our model is an extension to Grenadier's (1996), who emphasizes rational investment decisions possibly leading to oversupply in real estate markets, by further allowing for the important implication of irrational expectation for the strategic interaction amongst competing investors. In this model, two market participants are asymmetric because one of them is allowed to have heterogeneous expectations about the growth and volatility of demand shocks. Unlike most of previous studies that only simply think of this phenomenon as a result of irrationality, our model further finds that irrational investors' value-maximizing investment choices matter in understanding the strategic interaction of investment decisions in real estate markets, therefore providing additional insights into overbuilding and other puzzling phenomena in real estate markets.
\end{abstract}

KEYWORDS: Real options; Irrational expectations; Strategic investment; Overbuilding; Development cascade

SUPPLEMENTARY MATERIAL associated with this article can be found, in the online version, at http://dx.doi.org/10.3846/1648715X.2015.1073193

\section{INTRODUCTION}

Overbuilding is a noteworthy phenomenon in the evolution of real estate markets that can usually be characterized as a cyclical boom-and-bust pattern, and has attracted much attention from policy makers, market participants and researchers in the field of real estate. The recent serious crisis and sluggish recovery in the U.S. house industry even have further heightened our concerns over this phenomenon in real estate markets. For example, DeCoster and Strange (2012) highlight that there existed such category of phenomena in the US house market over the past several years, and attempt to provide an exploratory interpretation for this phenomenon from the angle of herding behavior. However, while a few interpretations for overbuilding can be identified from the relevant literature, the phenomenon is still thought of as a puzzle (Wang, Zhou 2000; Bar-Ilan, Strange 1996).

\footnotetext{
* Corresponding author. E-mail: fan10@konkuk.ac.kr
}

Among these interpretations, a real options-based analytical approach is appealing, in that it not only allows for an uncertain investment environment but also a rational expectation for real estate market (Grenadier 1996). Since overbuilding is of considerable importance to the academic research and practice of real estate markets, this paper attempts to develop a real options-based model to investigate the puzzling phenomenon in real estate markets by further incorporating heterogeneous expectations into this model as well as rational expectations. Our research sheds new light on the strategic implication of this phenomenon.

In the standard real options approach, an optimal investment opportunity is usually regarded as an American call option, and investors can endogenously choose the timing of their optimal investments based on the analytical framework of American options in order to maximize the values 
of their investment projects. Under this framework, an investment option should be exercised immediately when the value of the underlying state variable reaches the optimal investment threshold ${ }^{1}$. However, the practice of real-world investment competition suggests that optimal investment choices are usually not made in isolation but, to a large extent, affected by various strategic interactions among competing investors. As a consequence, recent literature has paid special attention to the important implications of these strategic interactions within the real options framework. For example, Williams (1993) provides an earlier exploration for equilibrium investment strategies of real estate developers within a real options framework, while these developers are rational and their options are exercised simultaneously. Grenadier (1996) further allows for the sequential exercise strategies of real estate developers. In Grenadier's model, two symmetric investors are assumed to hold common, rational expectations on the demand evolution of the real estate market. Grenadier's model discusses both sequential and simultaneous exercise equilibriums, and therefore provides an interesting explanation for the phenomena of development cascade and overbuilding in real estate markets. Grenadier (2002) further looks at competitive exercise strategies amongst multiple rational companies within a symmetric Nash equilibrium framework ${ }^{2}$. However, these existing studies typically assume that all market participants have the same, rational, expectations and that they all can process correctly all relevant information obtained without costs and therefore predict the stochastic evolution of an underlying state variable using an identical model. In reality, however, these assumptions might not be a good approximation to investment problems in the real world.

A great deal of literature has highlighted that investors' irrational expectations have important implications for strategic market competition (e.g., De Long et al. 1990, 1991; Kogan et al. 2006). In real estate literature, irrational expectations have been found to have significant impacts on the price fluctuations of real estate markets (e.g., Clayton 1996, 1997, 1998). More recent em-

\footnotetext{
${ }^{1}$ See Dixit and Pindyck (1994) and Trigeorgis (1996) for the detailed discussion on the standard real options models.

2 Smit and Trigeorgis (2004) provide a comprehensive survey of this category of literature on game-theoretic option models (see also Clapp et al. 2012).
}

pirical results have also shown that market participants might have irrational perspectives on the expected growth and volatility of real estaterelated variables such as market demand. For example, Glaeser et al. (2008) demonstrate that real estate bubbles might be, to a large extent, caused by the irrational market expectation deviating from rational market equilibrium. Mian and Sufi (2009) further provide evidence that the interaction of rational and excessive expectations for the U.S. housing market matters in interpreting the recent mortgage default crisis. More recently, Brueckner et al. (2012) highlight that mortgage lenders and borrowers could have different expectations on future house prices, which helps improve our understanding about the recent housing bubble in the U.S. As a result, our model extends the real options-based approach to examine the important implications of irrational expectations for the puzzling real estate phenomena of overbuilding and development cascade by allowing for irrational expectations on the growth and volatility of underlying demand variables in real estate markets. Development cascade is another puzzling phenomenon that has significant influence on real estate cycles and has also been discussed in Grenadier (1996). To our knowledge, little study has attempted to investigate the effects of irrational expectations on the strategic interaction amongst competing investors.

Furthermore, competing investors usually are not identical or homogeneous in the real world, so a few recent real options studies, such as Pawlina and Kort (2006), Kong and Kwok (2007), and Wang and Zhou (2006), have begun relaxing the strict assumption that these investors are completely the same. Among them, Wang and Zhou (2006) investigate optimal investment strategies in real estate markets allowing competitive developers to have different production capacities. Their findings clearly show that the differences of production capacities might delay these developers' expected exercise time, implying the importance of investigating asymmetric competition in the analysis of investment strategies ${ }^{3}$. Similar to these studies, we also take asymmetric competition into consideration, while we allow market participants to have different expectations on the growth and volatility of underlying state variables.

\footnotetext{
${ }^{3}$ See also Kong and Kwok (2007) and Pawlina and Kort (2006), who investigate the significant effects of asymmetry in both sunk costs and revenue flows on the strategic interaction of investment decisions in the duopoly context.
} 
The real estate development industry can be characterized as oligopolistic competition in many countries, because a few large powerful players play dominant roles in real estate development activities in these countries (e.g., Ong et al. 2003; Coiacetto 2009; Lai et al. 2004). Although duopoly, as a special form of oligopoly, might be seldom found in the real world, it is usually regarded as an ideal model for examining the essence of oligopolistic market structure. As a result, many studies such as Dixit and Pindyck (1994), Grenadier (1996) and Nielsen (2002) have examined the strategic interplay of competitive investors based on an option-based duopoly model where both duopolists are identical firms. Subsequent research has further extended these studies by relaxing one or several assumptions (e.g., Pawlina, Kort 2006; Kong, Kwok 2007; Mason, Weeds 2010). Similar to these subsequent studies, we also develop a duopoly model while we relax the assumption on rational expectation in order to shed new light on the phenomena of overbuilding and development cascade in real estate markets. We allow one agent to have irrational expectation on real estate demand, while the other still keeps the rational expectation. Such specification allows us to compare the differences between these two cases in order to highlight the important implications of irrational expectation.

Rational expectations can be understood in the sense that all relevant information can be obtained to forecast the future values of economic variables of interest, and such forecasting does not produce systematic errors deviating from the market equilibrium results (see, e.g., Sargent, Wallace 1976). Correspondingly, irrationality can be defined as deviations from the rational expectations. Irrational expectations might be caused by asymmetric or heterogeneous information, even though investors are of homogeneous priors. For example, De Long et al. (1990) show that different available information can result in two different categories of market participants, namely, rational arbitrageurs and irrational noise traders. These noise traders' beliefs drive the significant deviation of asset market prices from their fundamental values, and therefore matter in interpreting financial market anomalies. Garmaise and Moskowitz (2004) have demonstrated that some market participants hold superior information about the dynamics and conditions of real estate markets. Thus, our model assumes that two developers can form their rational expectations about the future demands for their respective buildings based on all relevant informa- tion, while they might collect limited information about their competitors. Specifically, one developer can form rational expectations regarding the future demand for his competitor's building as well as that for his own building. However, the other makes an irrational expectation on the future demand for his competitor's building, because this developer only holds limited information about his competitor ${ }^{4}$. As a result, both developers in our model have different expectations on the growth and volatility of underlying demand variables. We investigate the effects of the irrational expectations on these two developers' investment exercise strategies. Grenadier (1996) has shown that strategic market competition can result in the puzzling phenomena in real estate markets due to developers' investment-maximizing behaviors and the threat of preemption by their competitors. However, our findings show that if one developer misperceives the expected growth and volatility of the demand for his competitor's building and even if this developer follows the pattern of value-maximizing investment, this category of irrational expectations can still result in earlier or delayed investment and even excess supply in the real estate market. This suggests that "rational" choices of competitive investors with irrational expectations might distort strategic competition equilibriums in real estate markets, and therefore increase uncertainty in these markets.

Finally, real estate is a typical category of differentiated products, which are characterized by their heterogeneity and immobility such that no two properties are the same. Because of the differentiation of real estate in terms of location, structure or quality, real estate markets are usually found to be highly localized and segmented. Also, the differentiation of real estate is even often utilized by its developers as a competitive strategy. Thus, although the market demand for a certain category of buildings is the sum of their individual demands, the demands across these competing buildings might not be simply substituted due to their possible low substitution degree. If we use only one single geometric Brownian motion process to describe the demands for these buildings, this actually implies that these demands are driven only by one common market process. Shaked and Sutton (1982) stress that in a duopoly market two competing firms can reduce price competition and

\footnotetext{
${ }^{4}$ In effect, even though a developer holds only a set of incomplete information, he is likely to use it as though it was a set of complete information (Hong et al. 2007).
} 
realize positive profits at Nash equilibrium by producing differentiated products, therefore implying the importance of allowing for product differentiation in the competitive market. For this reason, we assume that the demands for two buildings in a duopoly context evolve following their respective geometric Brownian motions in order to take product differentiation into consideration. This suggests that common demand in this market can be captured by the instantaneous correlation between these two processes, whereas idiosyncratic demand caused by the differentiation of these two buildings may be described by the non-correlation components of these two processes.

The remainder of the paper is organized as follows. Section 2 proposes a basic model framework. Section 3 derives two developers' optimal investment thresholds and equilibrium exercise strategies in the benchmark setting. In Section 4, we take account of the effects of irrational expectations on equilibrium investment strategies. Section 5 conducts comparative static analysis. Section 6 draws relevant conclusions.

\section{MODEL SETTING}

Our model allows for a real estate market composed of two buildings, which are respectively owned by two individuals, denoted $i=1,2$. For simplicity, suppose that these two pre-existing buildings are obsolete. These two owners have opportunities to undertake development for their respective buildings in order to realize and enhance rental incomes from these two buildings. Suppose that developing a new building at any time will always incur a constant sunk cost $I>0$, while the time-to-build is not taken into our consideration ${ }^{5}$. We also assume that these two market agents are in competitive positions, and have to consider their competitors' development choices when deciding their own development strategies.

For the purpose of this study, we assume that these two agents might be of different expectations on market demand, while both of them are risk neutral and their redeveloped buildings have the same scale. Let $Q(t)$ represent the time- $t$ supply of the redeveloped buildings in the market such that $Q(t) \in(0,1,2)$. There exists a differentiable supply function $D[\cdot]$ satisfying $\partial D / \partial Q<0$, where $D(1)$

\footnotetext{
${ }^{5}$ Grenadier (1996) and Wang and Zhou (2006) have taken account of the time spent on the construction of buildings in their real options models. It can be shown that consideration of the time-to-build will not provide additional insights into our model results.
}

and $D(2)$ denote the supply conditions after only one building has been constructed and after both buildings have been developed completely, respectively.

Suppose that rent flows generated by these two buildings are determined by the following inverse demand function ${ }^{6}$ :

$$
P_{i}(t)=X_{i}(t) Y_{i}(t) D[Q(t)], i \in\{1,2\},
$$

where: $X_{i}(t)$ denotes the specific demand shock for agent 1's building, believed by owner $i$, whereas $Y_{i}(t)$ represents the demand shock for agent 2's building, believed by owner $i$. Such specification allows these two individuals to have heterogeneous perspectives on the demand processes. For expositional convenience, however, we specify that $X_{i}(t)$ evolves randomly following the same process for these two individuals in order to set up a benchmark case in this analysis. As a result, we can drop subscript $i$ from $X_{i}(t)$. Equation (1) can be rewritten as:

$$
P_{i}(t)=X(t) Y_{i}(t) D[Q(t)] .
$$

To consider the differentiation of these two buildings, the demand shock for agent 1's building is specified to be governed by the following geometric Brownian motion:

$$
d X=\mu X d t+\sigma X d B,
$$

where: $\mu$ and $\sigma$ are given positive constants, and $B$ denotes a standard Brownian motion. This specification implies that both agents have the same expectation on the growth and volatility of demand shocks for agent 1's building.

However, the demand shock for agent 2's building in agent $i$ 's perspective is believed to evolve in the following geometric Brownian motion:

$$
d Y_{i}=\alpha_{i} Y_{i} d t+c_{i} Y_{i} d W
$$

where: $W$ is another standard Brownian motion with the correlation $d B d W=\rho d t$, and $\rho<0$ represents the instantaneous correlation coefficient between $d B$ and $d W \cdot{ }^{7}$ In this equation, the constant $\alpha_{i}$ represents the percentage change rate of $Y_{i}(t)$

\footnotetext{
${ }^{6}$ Such specification closely follows Dixit and Pindyck (1994, Chapter 8), who define a similar functional form in considering two sources of demand uncertainty. Alternatively, we can also specify $X$ and $Y$ representing an industry-wide shock and firm-specific shock, respectively.

${ }^{7}$ Negative correlation, in effect, means negative externalities between two market agents, while positive correlation implies the contrary case. However, for simplicity, we only focus on the situation of negative externalities, whereas it is easy to extend our model to allow for positive externalities.
} 
expected by agent $i$, whereas the constant $c_{i}$ denotes the expected standard deviation, per unit of time, of $Y_{i}(t)$ in agent $i$ 's belief. Such specification allows both agents to have different expectations on the growth rate and volatility of demand shocks for agent 2's building, while the demand shock for this building in these two agents' perspectives is being driven by the same innovation process $W^{8}$.

\section{BENCHMARK SETTING AND INVESTMENT THRESHOLDS}

For ease of exposition, this section discusses a benchmark setting where both agents are assumed to have the same expectation on the growth and volatility of demand shocks for building 2. Similar to Kong and Kwok (2007), we take account of three categories of competitive equilibriums - preemptive exercise equilibrium, simultaneous exercise equilibrium and sequential exercise equilibrium. In other words, for any agent $i$, there are three possible strategies to choose in competitive equilibriums: he may undertake development for his pre-existing building earlier, as a leader, than his competitor; wait until his competitor has carried out a development action, as a follower; or exercise his development option with his competitor simultaneously. We solve the optimal decision problem for agent $i$ in the benchmark setting using a backward induction process as in a dynamic game. We first allow for the investment choice and threshold of his being a follower, and then take into account the case where this agent becomes a leader. When both agents develop their buildings simultaneously, it is easy to show that their respective investment option values will actually be the same as those of their being followers, respectively.

For expositional convenience, suppose that the other agent $j, j \in\{1,2\}$ but $j \neq i$, decides to become a leader and has begun developing her building first. In this case, agent $i$ will choose to be the follower and receive the duopoly rent of $X(t) Y_{i}(t) D(2)$ by (2) after exercising his development option. Let $F_{i}\left(X, Y_{i}\right)$ denote the value function of agent $i$ as the follower, and then his optimal problem can be expressed as:

\footnotetext{
8 The existing literature on real options has demonstrated that volatility is a major concern for investors in making investment decisions (e.g., Bulan et al. 2009), so our model also pays special attention to the important implications of expected distinct volatilities as well as expected different growth rates.
}

$$
\begin{aligned}
& F_{i}\left(X, Y_{i}\right)=\max _{T_{i f}^{*}} \mathrm{E}_{\mathrm{t}} \\
& {\left[\int_{T_{i f}^{*}}^{\infty} X(s) Y_{i}(s) D(2) e^{-r(s-t)} d s-e^{-r\left(T_{i f}^{*}-t\right)} I\right]^{\prime}}
\end{aligned}
$$

where: $T_{i f}^{*}$ denotes the follower's optimal investment time, and $r$ denotes the risk-free rate, which satisfies $r>\mu+\alpha_{i}+\rho \sigma c_{i}$ to ensure the exercise of his development option within a finite period of time.

For simplicity, let $X(t) Y_{i}(t)=\eta(t)$ where $\eta$ can be interpreted as the joint demand for these two buildings in the real estate market. A greater value of $\eta$ could indicate a larger joint demand for these two buildings. Since the follower faces the problem of choosing his optimal investment strategy, let $\eta_{i f}^{*}$ represent his optimal investment threshold. Following Dixit and Pindyck (1994, Chapter 6), we can obtain the following investment opportunity value for the follower (see Supplementary Appen$\operatorname{dix}$ A) 9 :

$$
F_{i}(\eta)= \begin{cases}\frac{I}{\beta_{i}-1}\left(\frac{\eta}{\eta_{i f}^{*}}\right)^{\beta_{i}}, & \text { if } \eta<\eta_{i f}^{*} \\ \frac{\eta D(2)}{r-\alpha_{i}-\mu-\rho \sigma c_{i}}-I, & \text { if } \eta \geq \eta_{i f}^{*}\end{cases}
$$

where: the constant $\beta_{i}$ is the positive root of the following quadratic equation ${ }^{10}$ :

$$
\begin{aligned}
& \left(\frac{1}{2} c_{i}^{2}+\frac{1}{2} \sigma^{2}+\rho \sigma c_{i}\right) \beta_{i}\left(\beta_{i}-1\right)+ \\
& \left(\alpha_{i}+\mu+\rho \sigma c_{i}\right) \beta_{i}-r=0 .
\end{aligned}
$$

The optimal investment threshold $\eta_{i f}^{*}$ is given by:

$$
\eta_{i f}^{*}=\frac{\beta_{i}}{\beta_{i}-1} \frac{I\left(r-\alpha_{i}-\mu-\rho \sigma c_{i}\right)}{D(2)}
$$

where: $\frac{\beta_{i}}{\beta_{i}-1}$ can be interpreted in terms of Tobin's $Q$. Since $\beta_{i}>1$, it is easy to show $\frac{\beta_{i}}{\beta_{i}-1}>1$. If $\eta<\eta_{i f}^{*}$, (6) suggests that agent $i$ will continue to wait for the optimal investment threshold $\eta_{i f}^{*}$.

\footnotetext{
${ }^{9}$ By Itô's Lemma, we can find that the stochastic process $X Y_{i}$ evolves in the form of $d X Y_{i}=\left(\mu+\alpha_{i}+\rho \sigma c_{i}\right) X Y_{i} d t+\sqrt{\sigma^{2}+c_{i}^{2}+2 \rho \sigma c_{i}} X Y_{i} d Z$, where $Z$ is a standard Brownian process. That is, $X Y_{i}$ follows a geometric Brownian motion. So, it is easy to show that the expected present value of the rent flow $X(t) Y_{i}(t) D(2)$ can be written as $\eta D(2) /\left(r-\alpha_{i}-\mu-\rho \sigma c_{i}\right)$.

${ }^{10}$ In equation (7), if both agents have different perspectives on the expected growth and standard deviation of demand shocks for agent 2's building, the two solutions of $\beta_{1}$ may be different from those of $\beta_{2}$.
} 
When $\eta$ reaches the threshold $\eta_{i f}^{*}$, it is optimal for this agent to exercise his option at once as a follower.

Conditional on the follower's optimal investment choice, we can further take the leader's investment strategies into account. In the case of preemptive exercise equilibrium, both agents have an incentive to take preemptive action to become a leader. That is, if neither of agents has obvious advantages in their competition, either of them is likewise likely to preempt the competition in order to obtain a first-mover advantage. Thus, the value of the investment opportunity, $L_{i}^{p}$, of a preemptive leader, say agent $i$, can be expressed as:

$$
\begin{aligned}
& L_{i}\left(X, Y_{i}\right)=E_{t}\left[\int_{T_{i l}}^{\infty} X(s) Y_{i}(s) D(1) e^{-r(s-t)} d s-e^{-r\left(T_{i l}-t\right)} I+\right. \\
& \left.\int_{T_{j f}^{*}}^{\infty} X(s) Y_{i}(s)[D(2)-D(1)] e^{-r(s-t)} d s\right],
\end{aligned}
$$

where: $T_{i l}$ is the leader's preemptive investment time.

Since our model allows both demand shocks to follow their respective Brownian motions, we can derive the following value function for the preemptive leader $i$ (see Supplementary Appendix A):

$$
L_{i}^{p}(\eta)= \begin{cases}\frac{D(1) \eta}{r-\alpha_{i}-\mu-\rho \sigma c_{i}}-I+\frac{D(2)-D(1)}{r-\alpha_{i}-\mu-\rho \sigma c_{i}} \eta_{j f}^{*}\left(\frac{\eta}{\eta_{j f}^{*}}\right)^{\beta_{i}}, & \text { if } \eta<\eta_{j f}^{*}, \\ \frac{D(2) \eta}{r-\alpha_{i}-\mu-\rho \sigma c_{i}}-I, & \text { if } \eta \geq \eta_{j f}^{*} .\end{cases}
$$

Given that the initial level of $\eta$ is sufficiently low, it is easy to understand that if being the follower for one agent will be more profitable, then this agent will not choose to enter the real estate market earlier as a leader. This implies that the preemptive investment threshold $\eta_{i p}^{*}$ should be determined by the relationship $L_{i}^{p}(\eta)=F_{i}(\eta)$. That is, at the preemptive threshold the agent will be indifferent between leading or following, while the preemptive investment time $T_{i l}$ might not be an optimal investment time. Specifically, if the initial level of $\eta$ is lower than $\eta_{i p}^{*}$, the preemptive leader will choose to wait until the threshold $\eta_{i p}^{*}$ is reached. If $\eta \geq \eta_{i p}^{*}$, the leader will adopt a preemptive action immediately to obtain the monopoly rent of $X(t) Y_{i}(t) D(1)$, thus generating a preemptive exercise equilibrium. However, when the follower enters this market, the leader will only receive the duopoly rent of $X(t) Y_{i}(t) D(2)$. The first line of equation (10) shows the preemptive leader's value function in these two cases. However, it is noteworthy that if the initial level of $\eta$ is higher than the follower investment threshold $\eta_{j f}^{*}$, both agents will exercise their development options immediately, resulting in a simultaneous exercise equilibrium.

In the case of sequential exercise equilibrium, one agent, say agent $i$, invests earlier as a dominant leader, while the other has no incentive to become the leader. The dominant leader's investment opportunity value, $L_{i}^{d}$, actually consists of two components, the monopoly option value and the negative impact of his competitor's entry into the market. Agent $i$ will undertake his development optimally at the moment the dominant threshold $\eta_{i d}^{*}$ is reached, and will receive the monopoly rent of $X(t) Y_{i}(t) D(1)$. The negative effect of the follower's option exercise on the leader's payoff is $X(t) Y_{i}(t)(D(2)-D(1))$. In this equilibrium, the value function of the dominant leader can be written as (see Supplementary Appendix A):

$$
L_{i}^{d}(\eta)=\left\{\begin{array}{l}
\frac{I}{\beta_{i}-1}\left(\frac{\eta}{\eta_{i d}^{*}}\right)^{\beta_{i}}+\frac{D(2)-D(1)}{r-\alpha_{i}-\mu-\rho \sigma c_{i}} \eta_{j f}^{*}\left(\frac{\eta}{\eta_{j f}^{*}}\right)^{\beta_{i}}, \\
\frac{D(1) \eta}{r-\alpha_{i}-\mu-\rho \sigma c_{i}}-I+\frac{D(2)-D(1)}{r-\alpha_{i}-\mu-\rho \sigma c_{i}} \eta_{j f}^{*}\left(\frac{\eta}{\eta_{j f}^{*}}\right)^{\beta_{i}}, \\
\text { if } \eta_{i d}^{*} \leq \eta<\eta_{j f}^{*}, \\
\frac{D(2) \eta}{r-\alpha_{i}-\mu-\rho \sigma c_{i}}-I, \\
\text { if } \eta \geq \eta^{*}
\end{array}\right.
$$

where:

$$
\eta_{i d}^{*}=\frac{\beta_{i}}{\beta_{i}-1} \frac{I\left(r-\alpha_{i}-\mu-\rho \sigma c_{i}\right)}{D(1)} .
$$

Expression (11) suggests that if the initial level of $\eta$ is smaller than $\eta_{i d}^{*}$, the dominant leader will not exercise his development option until the optimal investment threshold $\eta_{i d}^{*}$ is reached. If $\eta_{i d}^{*} \leq \eta<\eta_{j f}^{*}$, the dominant leader will exercise his investment option immediately to obtain the monopoly rent of $X(t) Y_{i}(t) D(1)$, resulting in a sequential exercise equilibrium. When the follower exercises her development option at the optimal threshold $\eta_{j f}^{*}$, the monopoly rent the leader receives will be reduced to the duopoly rent of $X(t) Y_{i}(t) D(2)$. In this case, the value of the dominant leader is actually the same as that of the preemptive leader, so their value functions have the same expression as shown in the first line of (10). However, if the initial level of $\eta$ is larger than $\max \left(\eta_{i f}^{*}, \eta_{j f}^{*}\right)$, both the leader and the follower will exercise their development options simultaneously, and a simultaneous exercise equilibrium will therefore happen.

Furthermore, we can derive the following proposition on preemptive investment. 
Proposition 1: Suppose that agents 1 and 2 have the same expectation on the growth and volatility of demand shocks for building 2 . If $\eta_{i f}^{*} \geq \eta_{j f}^{*}$, there must exist a preemptive threshold for agent $j$; However, if $\eta_{i f}^{*}<\eta_{j f}^{*}$, agent $i$ needs to allow for the preemptive action of agent $j$ if and only if the following inequality is satisfied:

$$
\frac{\eta_{j f}^{*}}{\eta_{i f}^{*}}<\left(\frac{x^{\beta_{j}}-1}{\beta_{j}(x-1)}\right)^{\frac{1}{\beta_{j}-1}},
$$

where:

$$
x=\frac{D(1)}{D(2)} .
$$

Proof: see Supplementary Appendix B.

This proposition allows for the existence conditions of asymmetric preemption. The left-hand side of inequality (13) represents both agents' follower threshold ratio. This suggests that when $\eta_{i f}^{*}<\eta_{j f}^{*}$ and if the follower threshold ratio satisfies the inequality (13), there must exist a preemptive investment threshold for agent $j$. If the follower threshold ratio is smaller, this means that agent $i$ 's follower threshold $\eta_{i f}^{*}$ relative to agent $j$ 's $\eta_{j f}^{*}$ increases, or vice verse $\eta_{j f}^{*}$ relative to $\eta_{i f}^{*}$ becomes smaller. The parameter $x$ reflects the relative supply conditions of the monopoly and duopoly states. When the preemptive threshold is crossed, agent $j$ may take a preemptive action immediately. However, if the inequality does not hold, the value of being a preemptive leader for this agent is lower than that of being a follower. The best strategic choice for agent $j$ is to wait until agent $i$ has undertaken his development and then exercise her option as the follower. However, if $\eta_{i f}^{*}$ is not smaller than $\eta_{j f}^{*}$, there always exists a preemptive threshold for agent $j$.

Further, we also have the following proposition concerning the preemptive and sequential exercise equilibrium.

Proposition 2: Suppose that agents 1 and 2 have the same expectation on the growth and volatility of demand shocks for building 2 . When $\eta_{i f}^{*}<\eta_{j f}^{*}$ and if the following inequality:

$$
\frac{\eta_{j f}^{*}}{\eta_{i f}^{*}}<\left(\frac{x^{\beta_{i}}-1}{\beta_{i}(x-1)}\right)^{\frac{1}{\beta_{i}-1}}
$$

holds, there respectively exist the preemptive thresholds for both agents $i$ and $j$. However, when $\eta_{i f}^{*}<\eta_{j f}^{*}$ and if the inequality:

$$
\frac{\eta_{j f}^{*}}{\eta_{i f}^{*}}>\left(\frac{x^{\beta_{j}}-1}{\beta_{j}(x-1)}\right)^{\frac{1}{\beta_{j}-1}}
$$

holds, agent $i$ is dominant in their competition and there therefore exists a sequential exercise equilibrium for both agents.

Proof: see Supplementary Appendix C.

According to Proposition 2, if inequality (14) holds, both agents $i$ and $j$ have their respective preemptive thresholds. This suggests that both agents' follower threshold ratio still plays an important role in determining the preemptive exercise equilibrium. Specifically, it can be shown that if $\eta_{i p}^{*}<\eta_{j p}^{*}$ and when the initial level of $\eta$ is sufficiently low, agent $i$ will begin development once $\eta_{i p}^{*}$ is reached, and agent $j$ is willing to be a follower and wait until $\eta_{j f}^{*}$ is reached. However, if the initial level of $\eta$ exceeds $\eta_{j f}^{*}$, the optimal strategy for both agents can be realized via their immediate simultaneous exercise. On the contrary, if $\eta_{i p}^{*}>\eta_{j p}^{*}$, agent $j$ will carry out a preemptive action the first moment that $\eta_{j p}^{*}$ is exceeded.

Furthermore, this proposition also specifies the existence conditions of sequential exercise equilibrium. Inequality (15) ensures not only the existence of $\eta_{i p}^{*}$ but also the absence of $\eta_{j p}^{*}$. That is, there is only one preemptive threshold for these two agents. In this case, agent $j$ will choose to wait until the agent $i$ has exercised his option, because the value of being the leader for agent $j$ will be smaller than the value of being the follower. This implies that agent $i$ has a dominant advantage in the competition relative to his competitor. If the initial level of $\eta$ is lower than $\eta_{i d}^{*}$, agent $i$ will exercise his option immediately once $\eta_{i d}^{*}$ is reached, and then agent $j$ will begin development when $\eta_{j f}^{*}$ is crossed. This means that both agents will carry out their developments according to a sequential exercise equilibrium. However, if the initial level of $\eta$ is larger than $\max \left(\eta_{i f}^{*}, \eta_{j f}^{*}\right)$, both agents will exercise their investment options simultaneously.

\section{HETEROGENEOUS BELIEFS IN DUOPOLY MARKET}

This section integrates heterogeneous expectations on the demand shocks for building 2 into the model developed above, where both the initial level of $\eta$ and the three types of investment thresholds have been identified to matter in determining our strategic exercise equilibriums. Similar to Kogan et al. (2006), the two market agents are assumed not to differ in their preferences but in their available information and demand expectations. Suppose 
that agent 2 holds all relevant information about the demand for his own building and can therefore form a rational expectation concerning its demand shocks. We therefore set $\alpha_{2}=\alpha$ and $c_{2}=c$ by dropping the subscript 2 for convenience. In contrast, however, we allow agent 1 to make an irrational expectation about her competitor's building demand utilizing her limited information about the demand shocks, therefore implying that $\alpha_{1}$ and $c_{1}$ might be different from $\alpha$ and $c$, respectively. We will discuss our model implications in two typical cases: $\alpha_{1} \neq \alpha$ and $c_{1}=c, \alpha_{1}=\alpha$ and $c_{1} \neq c$, respectively. Let $\eta_{1 f}^{* *}, \eta_{1 p}^{* * *}$, and $\eta_{1 d}^{* * *}$ denote agent 1 's follower, preemptive and dominant investment threshold under the parameters $\alpha_{1}$ and $c_{1}$, respectively. That is, they all represent the investment thresholds driven by agent 1's irrational expectations. As for the case of $\alpha_{1} \neq \alpha$ and $c_{1}=c$, we can obtain the following proposition.

Proposition 3: Suppose that agents 1 and 2 have different expectations on the growth of demand shocks for agent 2's building, while they still have the same expectation on the volatility of the shocks. For $\eta_{1 f}^{* * *}<I \beta_{1} \sqrt{\Delta_{1}} / D(2)$, if $\alpha_{1}>\alpha$, then agent 1's optimal follower threshold satisfies the relationship $\eta_{1 f}^{* * *}<\eta_{1 f}^{*}$, and vice versa ${ }^{11}$. However, if $\eta_{1 f}^{* * *}>I \beta_{1} \sqrt{\Delta_{1}} / D(2), \alpha_{1}<\alpha$ can also lead to $\eta_{1 f}^{* *}<\eta_{1 f}^{*}$, and vice versa.

Proof: see Supplementary Appendix D.

Proposition 3 shows that given $\eta_{1 f}^{* * *}<I \beta_{1} \sqrt{\Delta_{1}} / D(2)$, when agent 1 has a higher expectation on the growth of demand shocks for agent 2's building than expected rationally by agent 2 , this agent will have a lower follower investment threshold than agent 2 rationally expects. In other words, if the irrational expectation on the demand growth of the building is taken into account and if the initial level of $\eta$ is sufficiently low, this agent might exercise his development option earlier as the follower than expected rationally by agent 2 . This implies that the irrational expectation matters and might speed up the follower's investment action ${ }^{12}$.

${ }^{11}$ It is noteworthy that in this proposition, $\beta=\beta_{2}$ and $\Delta=\Delta_{2}=\left(\alpha_{2}+\mu-\frac{1}{2} \sigma^{2}-\frac{1}{2} c_{2}^{2}\right)^{2}+2 r\left(\sigma^{2}+c_{2}^{2}+2 \rho \sigma c_{2}\right)$.

We drop the subscript 2 from these two variables in that agent 2's perspective on the expected growth is determined by the real market conditions.

${ }^{12}$ Since $X Y$ follows a geometric Brownian motion as shown earlier, by Wong (2007) and Lin (2006, Chapter 4) one can find that agent 1's expected time to exercise her option relative to an investment threshold such as $\eta_{1 f}^{* *}$ can be expressed as
On the contrary, it is easy to understand that if $\alpha_{1}<\alpha$, agent 1 would have a higher follower threshold than agent 2 rationally expects. That is, if agent 1 expects a lower growth of demand shocks for agent 2's building and if the initial level of $\eta$ is sufficiently low, agent 1 might undertake her development later than agent 2 rationally expects. In other words, the irrational expectation might make the follower postpone her investment.

The previous section has identified three categories of competitive equilibriums. For these three equilibriums, it can be shown by Propositions 1 and 2 that agent 1's entry thresholds should satisfy the following order of relative magnitude:

$$
\eta_{1 p}^{*}<\eta_{1 d}^{*}<\eta_{1 f}^{*} \text {. }
$$

When the effect of irrational expectation is taken into consideration, (16) can be rewritten as:

$$
\eta_{1 p}^{* *}<\eta_{1 d}^{* *}<\eta_{1 f}^{* *} \text {. }
$$

Inequalities (16) and (17) suggest that agent 1 's dominant investment threshold be lower than her follower investment threshold, otherwise this agent will exercise her option as the follower when the follower threshold is reached first. These inequalities also indicate that agent 1's preemptive threshold should in turn be smaller than her dominant investment threshold. It is easy to understand that if this agent's dominant threshold is lower her preemptive threshold, she will not wait until a preemptive threshold is reached, because her dominant threshold is the optimal threshold in the duopoly context. This also implies the absence of the preemptive threshold in this case.

Thus, if the initial level of $\eta$ is sufficiently low, one agent would preempt the competition first when the other has no obvious competitive advantage, thus resulting in a preemptive exercise equilibrium. If this agent has succeeded in taking a preemptive action, the other will have to wait until the optimal follower threshold is reached. In this case, we can obtain the following proposition.

Proposition 4: Suppose that agents 1 and 2 have different expectations on the growth of demand shocks for agent 2's building, while they still have the same expectation on the volatility of the

$E\left(T_{1 f}^{* * *}\right)=\ln \left(\eta_{1 f}^{* * *} / \eta(0)\right) /\left[\mu+\alpha_{1}-\left(\sigma^{2}+c_{1}^{2}\right) / 2\right]$. Without loss of generality, suppose that $\mu+\alpha_{1}>\left(\sigma^{2}+c_{1}^{2}\right) / 2$ always holds. It is easy to find that given $\eta_{1 f}^{* * *}<I \beta_{1} \sqrt{\Delta_{1}} / D(2)$, an increase in $\alpha_{1}$ can reduce the numerator $\ln \left(\eta_{1 f}^{* *} / \eta(0)\right)$ but raise the denominator $\left[\mu+\alpha_{1}-\left(\sigma^{2}+c_{1}^{2}\right) / 2\right]$, therefore accelerating this agent's expected exercise time, all else being equal, and vice versa. 
shocks. Given that both $\eta_{1 f}^{*}$ and $\eta_{1 f}^{* * *}$ are lower than $\eta_{2 f}^{*}$, if $\alpha_{1}>\alpha$, then $\eta_{1 p}^{* *}<\eta_{1 p}^{*}$, and vice versa.

Proof: see Supplementary Appendix E.

Given that the initial level of $\eta$ is sufficiently low, this proposition suggests agent 1 might have a lower preemptive threshold than expected rationally by agent 2 , if this agent's expectation satisfies $\alpha_{1}>\alpha$. In other words, the irrational expectation on the growth of demand shocks might distort this agent's behavior in the real estate market and further speed up or delay the supply of this market.

However, it is noteworthy that Grenadier (1996) defines overbuilding as the recession-induced construction boom in the simultaneous exercise equilibrium. Even though both agents are identical and symmetric in a duopoly context, Grenadier find that strategic market competition can result in the overbuilding phenomenon in the face of plummeting demand of real estate markets, due to their avoiding the problem of preemption. Similar to Grenadier (1996), to examine this phenomenon we suppose that the initial level of $\eta$ is greater than both agents' follower thresholds, and we can therefore take account of a category of equilibriums where one agent will exercise the option to develop immediately as soon as the demand $\eta$ falls to $\max \left(\eta_{1 f}^{* *}, \eta_{1 f}^{*}\right)$ . It is easy to show that if $\eta_{1 f}^{* *}>\eta_{1 f}^{*}>\eta_{2 f}^{*}$ and when $\eta$ drops to the follower threshold $\eta_{1 f}^{* *}$, agent 1's option exercise will happen earlier than expected rationally by her competitor owing to fear of being preempted. ${ }^{13}$ The inequality $\eta_{1 f}^{* *}>\eta_{1 f}^{*}>\eta_{2 f}^{*}$ indicates that agent 2's follower investment threshold is lower than agent 1's rational follower threshold, which is in turn smaller than her follower threshold driven by an irrational demand expectation. This implies that when facing the falling market demand, the irrational expectation can also speed up the supply of the real estate market. That is to say, it might also lead to the overbuilding phenomenon in the market ${ }^{14}$.

$\overline{13}$ It is noteworthy that by Lin (2006: 127 , Chapter 4), since the initial demand level has exceeded the follower investment threshold in this case, agent 1's expected time to exercise the option of investment can be obtained as $E\left(T_{1 f}^{* * *}\right)=\ln \left(\eta(0) / \eta_{1 f}^{* *}\right) /\left[\mu+\alpha_{1}-\left(\sigma^{2}+c_{1}^{2}\right) / 2\right]$. According to proposition 3 , if $\eta_{1 f}^{* * *}<I \beta_{1} \sqrt{\Delta_{1}} / D(2)$, one may find that $\partial E\left(T_{1 f}^{* *}\right) / \partial a_{1}>0$ can be satisfied, and a decrease in $\alpha_{1}$ therefore can speed up agent 1's expected exercise time.

${ }^{14}$ It is noteworthy that when real estate markets are perfectly competitive, the potential of RCBs will disappear as shown in Grenadier (1996). However, real estate markets can be characterized as oligopolistic competition in many countries, because a few large powerful developers play dominant roles in real estate development activities in these countries (e.g., Ong
In contrast, regarding the case of $c_{1} \neq c$ and $\alpha_{1}=\alpha$, we have the following proposition.

Proposition 5: Suppose that agents 1 and 2 have the same expectation on the growth of demand shocks for agent 2's building, while they have different expectations on the volatility of the shocks. For $c_{1}>\frac{\rho \sigma \beta_{1}}{1-\beta_{1}}$, if $c_{1}<c$, agent 1's follower threshold satisfies $\eta_{1 f}^{* *}<\eta_{1 f}^{*}$, and vice versa.

Proof: see Supplementary Appendix F.

This proposition suggests that if agent 1 believes that there is a smaller volatility of demand shocks for agent 2's building, and if $c_{1}>\frac{\rho \sigma \beta_{1}}{1-\beta_{1}}$, this agent will have a lower follower threshold than expected rationally by agent 2 . If the initial level of $\eta$ is sufficiently low as well, agent 1 might prefer to exercise her option earlier as the demand approaches $\eta_{1 f}^{* * *}$, because a smaller volatility $c_{1}$ can reduce $E\left(T_{1 f}^{* * *}\right)$ given in footnote 12 . This implies that an irrational expectation on the volatility might also distort this agent's investment decisions and therefore hasten the supply in the real estate market.

However, if the initial level of $\eta$ is higher than agent 1's follower threshold $\eta_{1 f}^{* * *}$, this agent is also likely to exercise her development option earlier to avoid being preempted than expected rationally by her competitor as the demand gradually drops to the follower threshold $\eta_{1 f}^{* *}$. We still consider the case of $\eta_{1 f}^{* *}>\eta_{1 f}^{*}>\eta_{2 f}^{*}$, implying that agent 1's follower threshold $\eta_{1 f}^{* *}$ driven by an irrationally expected demand volatility is the highest among these three follower thresholds. This suggests that we can consider again the set of equilibrium strategies that agent 1 preempts her rival when the demand $\eta$ falls to the follower threshold $\eta_{1 f}^{* *}$. In this case, fear of being preempted can make agent 1 exercise earlier the option to develop than expected rationally, therefore leading to the oversupply of real estate following the fall in the market demand. ${ }^{15}$ This

et al. 2003; Coiacetto 2009; Lai et al. 2004). Coiacetto (2009) systematically addresses the major reasons why many real estate markets are dominated by a few large developers. Thus, our findings on overbuilding can still provide new insights into the effects of irrational expectations on real estate development activities and have important implication for the real estate development industry.

${ }^{15} \mathrm{As}$ shown in footnote 13, agent 1 's expected exercise time in this case can be obtained as $E\left(T_{1 f}^{* *}\right)=\ln \left(\eta(0) / \eta_{1 f}^{* *}\right) /\left[\mu+\alpha_{1}-\left(\sigma^{2}+c_{1}^{2}\right) / 2\right]$. Thus, according to Proposition 5, one may find that given $c_{1}>\frac{\rho \sigma \beta_{1}}{1-\beta_{1}}, \partial E\left(T_{1 f}^{* *}\right) / \partial c_{1}<0$ can be met, and an increase in $c_{1}$ therefore can raise this agent's investment threshold and in turn accelerate his expected exercise time. 
implies that an irrationally expected demand volatility may also result in the phenomenon of overbuilding in the market.

In addition, let $\eta_{1 d}^{* *}$ and $\eta_{1 d}^{*}$ be the dominant thresholds under the parameters $\alpha_{1}$ and $c_{1}$ and under the parameters $\alpha$ and $c$, respectively. We have the following proposition on the sequential exercise equilibrium.

Proposition 6: Suppose that agents 1 and 2 have different expectation on the growth of demand shocks for agent 2's building, while they still have the same expectation on the volatility of the shocks. For $\eta_{1 d}^{* *}<I \beta_{1} \sqrt{\Delta_{1}} / D(1)$, if $\alpha_{1}>\alpha$, then agent 1's dominant investment threshold satisfies the relationship $\eta_{1 d}^{* *}<\eta_{1 d}^{*}$, and vice versa. However, if $\eta_{1 d}^{* * *}>I \beta_{1} \sqrt{\Delta}_{1} / D(1), \alpha_{1}>\alpha$ can make $\eta_{1 d}^{* * *}>\eta_{1 d}^{*}$ hold, and vice versa.

Proof: see Supplementary Appendix G.

In the case of sequential exercise equilibrium, due to her competitive advantage, agent 1 chooses to exercise the development option at the optimal dominant threshold, whereas her competitor chooses to become the follower. In Proposition $6, \eta_{1 d}^{* * *}<\eta_{1 d}^{*}$ indicates that should agent 1 be the dominant leader, she will have a lower dominant investment threshold than expected rationally by agent 2 . That is, if agent 1 has a higher expectation on the demand growth for agent 2's building and when $\eta_{1 d}^{* *}<I \beta_{1} \sqrt{\Delta_{1}} / D(1)$, this agent's dominant threshold will be smaller than agent 2 expects rationally. When the initial level of $\eta$ is sufficiently low, this suggests that agent 1 might begin development earlier in the sequential equilibrium than expected rationally by agent 2 , thus accelerating the supply in the real estate market.

On the other hand, however, Proposition 6 also suggests one contrary case. If agent $1 \mathrm{ex}$ pects a higher growth of demand shocks for agent 2's building but when $\eta_{1 d}^{* *}>I \beta_{1} \sqrt{\Delta_{1}} / D(1)$, this agent would choose a higher dominant investment threshold than expected rationally by agent 2 . These results imply that if the initial level of $\eta$ is sufficiently low, a higher expectation on the demand growth might also delay the supply of the real estate market.

However, it is worth noting that strategic market competition can result in development cascades in real estate markets as well. Grenadier (1996) defines the development cascade as a quick succession of development actions. In a sequential exercise equilibrium, strategic competition can make the follower invest earlier, implying the phenomenon of development cascade. In our case, if agent 2 is the dominant leader, agent 1 can only choose to develop as the follower in the sequential equilibrium. Thus, according to discussion associated with propositions 3 and 5 , this suggests that if agent 1 has a higher expected demand growth or a smaller expected demand volatility for agent 2's building than expected rationally by agent 2 , this agent might have a lower follower threshold, thus exercising her development option earlier than expected rationally ${ }^{16}$. This implies that irrational expectation on real estate demand also probably results in development cascade. In other words, development cascades can also be alternatively explained by irrational market expectation.

In contrast, in the case of $c_{1} \neq c$ and $\alpha_{1}=\alpha$, We have the following proposition on the dominant equilibrium.

Proposition 7: Suppose that agents 1 and 2 have the same perspective on the expected growth of demand shocks for building 2 , while they have heterogeneous beliefs on the volatility of the demand shocks. Given $c_{1}>\frac{\rho \sigma \beta_{1}}{1-\beta_{1}}$, if $c_{1}>c$, there must be $\eta_{1 d}^{* *}<\eta_{1 d}^{*}$, and vice versa.

Proof: see Supplementary Appendix H.

Similarly to Proposition 6, Proposition 7 also discusses the case associated with the sequential exercise equilibrium, while it pays special attention to the effect of irrational expectations pertinent to the volatility of demand shocks for agent 2's building. In effect, this proposition, coupled with Proposition 4, shows that whether agent 1 is a dominant leader or a follower, and if $c_{1}>c$ and $c_{1}>\frac{\rho \sigma \beta_{1}}{1-\beta_{1}}$, this agent will have lower investment thresholds in these two cases than expected rationally by agent 2 . These results suggest that irrational expectations on the demand volatility might not only cause a wait-and-see mood in real estate markets, but also lead to development cascades in these markets.

16 In Grenadier (1996), to examine the development cascade, the initial demand level is assumed to begin at a leader's investment threshold. Similarly, letting the initial demand level start at $\eta_{2 d}^{*}$, it can be shown that by Wong (2007) and Lin (2006, Chapter 4), agent 1's expected time to exercise the option of investment as a follower can be expressed a s $E\left(T_{1 f}^{* * *}\right)=\ln \left(\eta_{1 f}^{* *} / \eta_{2 d}^{*}\right) /\left[\mu+\alpha_{1}-\left(\sigma^{2}+c_{1}^{2}\right) / 2\right], \quad$ where $\eta_{1 f}^{* *}>\eta_{2 d}^{*}$. However, since $\eta_{2 d}^{*}$ is not associated with both $\alpha_{1}$ and $c_{1}$, it is easy to find, as discussed previously, that an increase in $\alpha_{1}$ or a decrease in $c$ can speed up this agent's expected exercise time, all else being equal. 


\section{NUMERICAL ANALYSIS}

Irrational expectations might lead to deviations of real estate markets from their market fundamentals. Clayton $(1996,1997,1998)$ show many realistic examples on these effects. To better examine the effects of irrational expectations, this section further investigates numerically how changes in two crucial parameters reflecting irrational expectations, $\alpha_{1}$ and $c_{1}$, affect the investment thresholds and optimal strategies of market participants in the duopoly market. In other words, we attempt to consider a more realistic real estate example where developers have varying irrational expectations on the growth and volatility of real estate markets. We choose the input parameter values to reflect the real estate market practice as closely as possible. As discussed above, we have identified that the impacts of changes in $\alpha_{1}$ on agent 1 's dominant and follower investment thresholds are ambiguous. This is because all the two thresholds consist of two components. For example, $\eta_{1 f}^{* *}$ is composed of the Tobin's $Q$ ratio of $\beta_{1} /\left(\beta_{1}-1\right)$ and the term of $I\left(r-\alpha_{1}-\mu-\rho \sigma c_{1}\right) / D(2)$. We can easily find that an increase in $\alpha_{1}$ raises the value of the Tobin's $Q$ but reduces the value of $I\left(r-\alpha_{1}-\mu-\rho \sigma c_{1}\right) / D(2)$. This implies that the effects of changes in $\alpha_{1}$ on these investment thresholds are determined by the interaction of these two opposite forces.

In particular, Figure 1 also plots the impacts of increasing $\alpha_{1}$ on all three investment thresholds. It is shown that all these three thresholds drop with the increase of $\alpha_{1}$ in the range from 0 to 0.025 . Also, among these three thresholds, the variation of $\eta_{1 p}^{* *}$ is observed to be largest, implying that agent 1's preemptive threshold is most sensitive to changes in the irrationally expected demand growth.

Similarly, we can find that the impacts of changes in $c_{1}$ on the agent 1's dominant and follower investment thresholds are ambiguous as well, regardless of being a follower or a leader. However, Supplementary Appendix F also shows that when the condition $c_{1}>\frac{\rho \sigma \beta_{1}}{1-\beta_{1}}$ is satisfied, we can find $\partial \eta_{1 f}^{* *} / \partial c_{1}>0$ holds. Similarly, according to Supplementary Appendix $\mathrm{H}$ we can find that if $c_{1}>\frac{\rho \sigma \beta_{1}}{1-\beta_{1}}$, there must be $\partial \eta_{1 d}^{* *} / \partial c_{1}>0$. Further, Figure 2(I) shows that when $\rho=-0.5$ and $c_{1}$ increases from 0.001 to 0.17 , these two thresholds drop in $c_{1}$ first and then rise gradually, exhibiting a $\mathrm{U}$ shaped pattern. Also, we can identify the convexity of $\eta_{1 p}^{* * *}$ as $c_{1}$ increases. In particular, among these

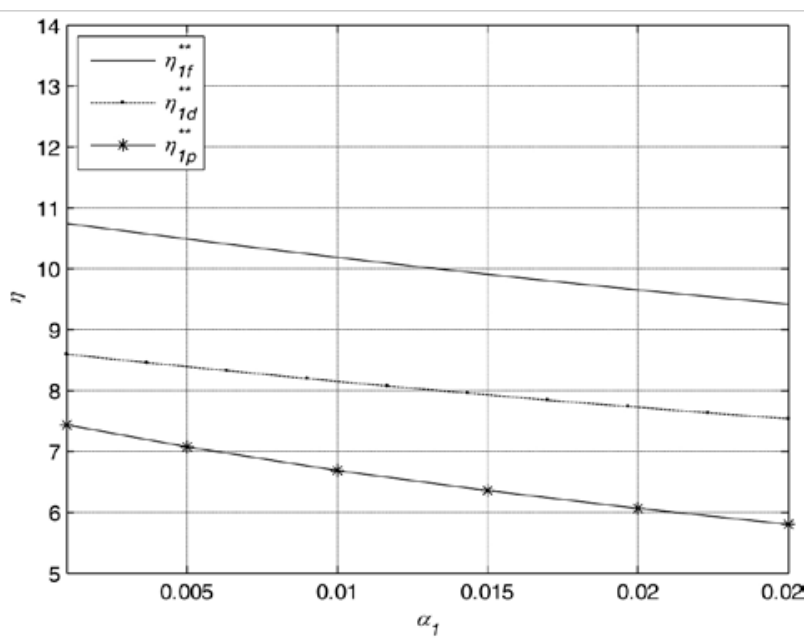

Fig. 1. Impacts of $\alpha_{1}$ on investment thresholds of agent 1

Note: All the parameter values are set as follows: $r=$ $0.05, I=100, \rho=-0.1, D(1)=1, D(2)=0.8, \mu=0.02, \alpha_{j}=$ $0.01, \sigma=0.2, c_{i}=0.1, c_{j}=0.1$

three thresholds, the variation of $\eta_{1 p}^{* *}$ is found to be greatest as $c_{1}$ increases in the same range. This implies that agent 1's preemptive threshold is most sensitive to changes in the irrationally expected demand volatility. However, when the absolute value of $\rho$ becomes smaller and, specifically, $\rho=-0.1$, Figure 2(II) demonstrates that all these three thresholds gradually increase with the increase of $c_{1}$.

Finally, due to the important implication of the correlation $\rho$ in our numerical analysis, we also derive the comparative static results with respect to $\rho$ :

$$
\frac{\partial \eta_{1 f}^{* *}}{\partial \rho} \frac{<}{<} 0, \quad \frac{\partial \eta_{1 d}^{* *}}{\partial \rho} \frac{>}{<} 0 .
$$

Although the impacts of changes in $\rho$ on the agent 1's dominant and follower investment thresholds are ambiguous, Supplementary Appendix J shows that $\eta_{1 f}^{* *}$ and $\eta_{1 d}^{* *}$ will increase with the correlation when $\eta_{1 f}^{* *}>I \sqrt{\Delta_{1}} / D(2)$ holds. This indicates that higher correlation might delay or speed up investment exercise in these two cases.

Figure 3 provides further numerical evidence about these effects of varying correlation. Also, we find that among all these three thresholds, the variation of $\eta_{1 f}^{* * *}$ is largest when correlation varies in the same range, therefore implying the most sensitivity of $\eta_{1 f}^{* *}$ to changes in $\rho$. However, the variation of $\eta_{1 p}^{* * *}$ is found to be smallest as $\rho$ changes within the same range. These results are different from the impacts of those two other parameters identified earlier. Higher correlation raises the values of $\eta_{1 f}^{* * *}$ and $\eta_{1 d}^{* * *}$, and therefore 
(l)

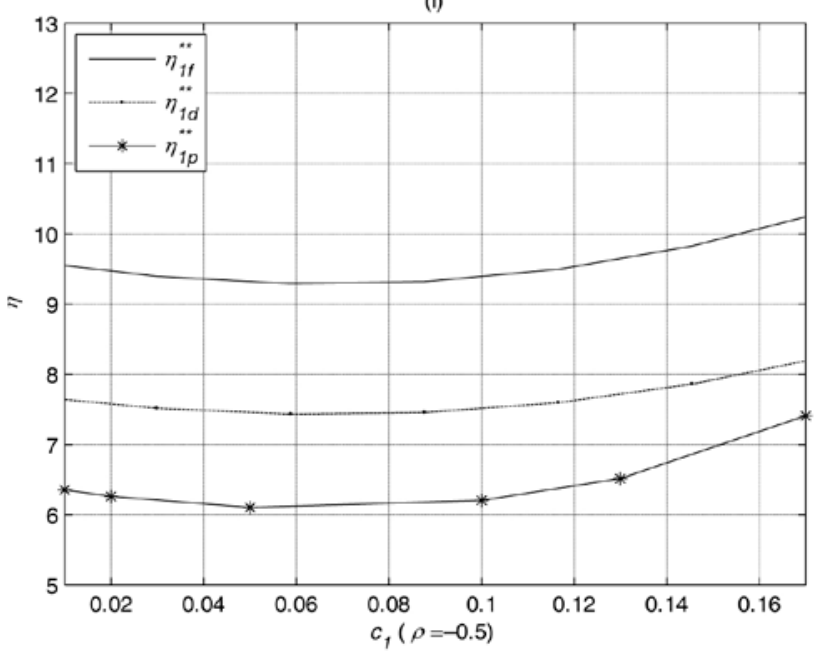

(II)

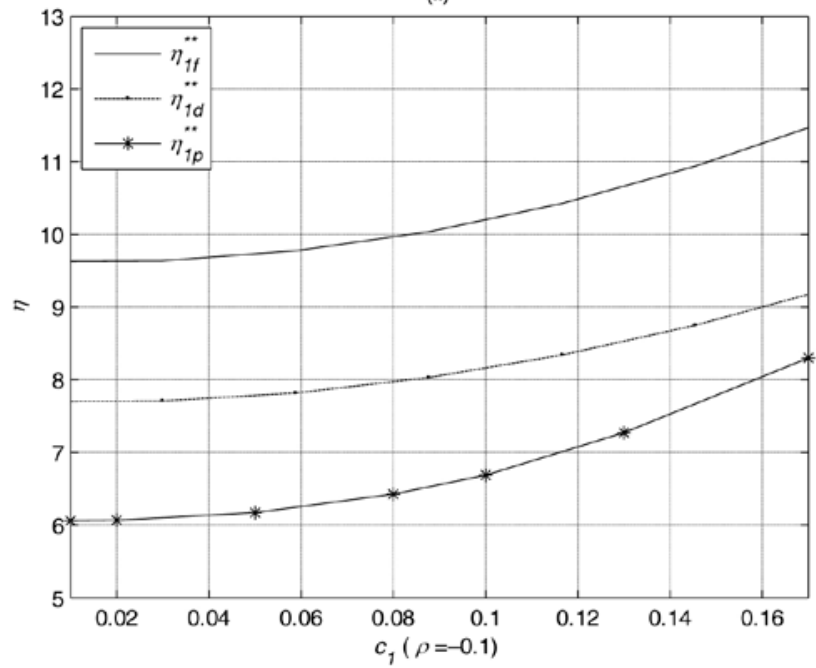

Fig. 2. Impacts of $c_{1}$ on investment thresholds of agent 1

Note: All the parameter values are set as follows: $r=0.05$, $I=100, D(1)=1, D(2)=0.8, \mu=0.02, \alpha_{i}=0.01, \alpha_{j}=0.01$, $\sigma=0.2$, and $c_{j}=0.1$.

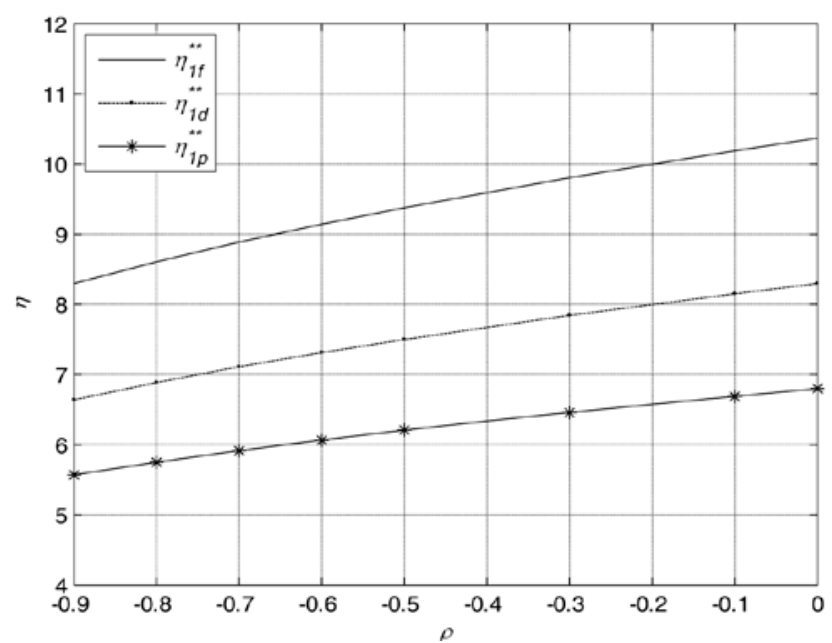

Fig. 3. Impacts of $\rho$ on investment thresholds of agent 1

Note: All the parameter values are set as follows: $r=$ $0.05, I=100, D(1)=1, D(2)=0.8, \mu=0.02, \alpha_{i}=0.01, \alpha_{j}=$ $0.01, \sigma=0.2, c_{i}=0.1, c_{j}=0.1$. lowers agent 1's investment enthusiasm in these two cases if the initial level of demand is sufficiently low. For preemption, however, $\eta_{1 p}^{* * *}$ experiences the smallest change as $\rho$ varies, because this agent tends to obtain a first-mover advantage in a successful preemptive action and the leadership in the competition.

\section{CONCLUSIONS AND DISCUSSIONS}

This paper investigates the effects of heterogeneous expectation on the strategic interaction of investment decisions between two competitive real estate developers in a duopoly market. Our gametheoretic option model is an extension to Grenadier (1996) by allowing these two developers to have different expectations on the growth and volatility about market demand. Our results show that these heterogeneous expectations have important implications for the phenomena of overbuilding and development cascade in real estate markets.

Our model considers three categories of competitive equilibriums, for which we derive the competitive investors' optimal investment thresholds and their investment option values. The previous literature such as Grenadier (1996) has demonstrated that strategic market competition can force rational market participants to hasten their investment exercise, therefore probably resulting in overbuilding and development cascades in real estate markets. We find that irrational expectations on the growth and volatility of market demand might further make investors' development strategies deviate from the equilibrium exercise strategies predicted by the standard game-theoretic option model. These deviations can lower or enhance irrational investors' market entry thresholds, therefore increasing uncertainty in real estate markets. In particular, even though investors are of irrational expectations, we still have reasons to believe that they still tend to maximize their investment project values. As a result, irrational investors' value-maximizing investment choices are found to matter in understanding the equilibrium market strategies in real estate markets, and might further hasten their development activities and aggravate the excess supply conditions in these markets. Hence, our model provides another testable explanation about the development cascade and overbuilding phenomena.

Furthermore, we find that among the three categories of investment thresholds, preemptive thresholds are more sensitive to changes in irrational expectations than the other categories of 
investment thresholds. The correlation coefficient between different demand shocks is also found to play an important role in determining these three investment thresholds, while the preemptive thresholds under irrational expectation are less sensitive to changes in the correlation than those other thresholds. Also, it is noteworthy that although our model is developed via allowing for strategic development decisions affected by irrational expectations in real estate markets, our model results can also be likewise applied to investigate oversupply issues in other industries.

Last but not least, our research does not attempt to suggest that real estate market equilibriums are driven by irrational expectations. In our benchmark setting, we actually still assume that two market agents have rational expectations in three competitive equilibriums, thus implying that our equilibriums are built on the basis of rational expectations. In our model, since rational expectations provide a foundation for deriving equilibrium exercise strategies, this implies that rational expectations still matter in driving the equilibriums of real estate markets in our analysis. Despite that, we have further noticed that market participants might have irrational expectations, which can generate effects on real estate markets and their cycles. We emphasize here that irrational expectations might cause real estate markets to deviate from their equilibriums and even aggravate development cascade and overbuilding. This suggests that real estate markets still might be brought back by market forces to their equilibriums from the deviations if the expectations are corrected. Clayton (1997, 1998) demonstrates that anomalies in real estate markets can partly be caused by irrational expectations. Other studies, such as Hamilton and Schwab (1985), Capozza and Seguin (1996) and Meese and Wallace (1994), also provide empirical evidence against rational expectations on real estate markets. These results imply the importance of irrational expectations in understanding real estate cycles.

\section{ACKNOWLEDGMENT}

We would like to thank Russell W. Cooper, Kerry D. Vandell, Yi Wen, Jian Yang, Lin Zhang, Weida Kuang and seminar participants at the 2011 Asian Real Estate Society-American Real Estate and Urban Economics Society Joint International Conference, and Southwestern University of Finance and Economics for helpful comments. This research was supported by the National Social Science Fund of China (No: 14BJY180) and the Chongqing Social Science Planning Project (No. 2013QNJJ16). Any errors are our own.

\section{REFERENCES}

Bar-Ilan, A.; Strange, W. C. 1996. Investment lags, American Economic Review 86(3): 610-622.

Brueckner, J. K.; Calem, P. S.; Nakamura, L. I. 2012. Subprime mortgages and the housing bubble, Journal of Urban Economics 71: 230-243. http://dx.doi. org/10.1016/j.jue.2011.09.002

Bulan, L.; Mayer, C.; Somerville, C. T. 2009. Irreversible investment, real options and competition: evidence from real estate development, Journal of Urban Economics 65: 237-251. http://dx.doi.org/10.1016/j. jue.2008.03.003

Capozza, D.; Seguin, P. 1996. Expectations, efficiency and euphoria in the housing market, Regional Science and Urban Economics 26: 369-386. http://dx.doi. org/10.1016/0166-0462(95)02120-5

Clapp, J. M.; Bardos, K. S.; Wong, S. K. 2012. Empirical estimation of the option premium for residential redevelopment, Regional Science and Urban Economics 42: 240-256. http://dx.doi.org/10.1016/j.regsciurbeco.2011.08.007

Clayton, J. 1996. Rational expectations, market fundamentals and housing price volatility, Real Estate Economics 24(4): 441-470. http://dx.doi. org/10.1111/1540-6229.00699

Clayton, J. 1997. Are housing price cycles driven by irrational expectations?, Journal of Real Estate Finance and Economics 14(3): 341-363. http://dx.doi. org/10.1023/A:1007766714810

Clayton, J. 1998. Further evidence on real estate market efficiency, Journal of Real Estate Research 15: 41-57.

Coiacetto, E. 2009. Industry structure in real estate development: is city building competitive?, Urban Policy and Research 27(2): 117-135. http://dx.doi. org/10.1080/08111140802499080

De Long, J. B.; Shleifer, A.; Summers, L. H.; Waldmann, R. J. 1990. Noise trader risk in financial markets, Journal of Political Economy 98: 703-378. http://dx.doi.org/10.1086/261703

De Long J. B.; Shleifer, A.; Summers, L. H.; Waldmann, R. J. 1991, The survival of noise traders in financial markets, Journal of Business 64(1): 1-19. http://dx.doi.org/10.1086/296523

DeCoster, G. P.; Strange, W. C. 2012. Developers, herding, and overbuilding, Journal of Real Estate Finance and Economics 44: 7-35. http://dx.doi.org/10.1007/ s11146-011-9309-0

Dixit, A. K.; Pindyck, R. S. 1994. Investment under uncertainty. Princeton, NJ: Princeton University Press.

Garmaise, M. J.; Moskowitz, T. J. 2004. Confronting information asymmetries: evidence from real estate markets, Review of Financial Studies 17(2): 405-437. http://dx.doi.org/10.1093/rfs/hhg037 
Glaeser, E. L.; Gyourko, J.; Saiz, A. 2008. Housing supply and housing bubbles, Journal of Urban Economics 64: 198-217. http://dx.doi.org/10.1016/j. jue.2008.07.007

Grenadier, S. R. 1996. The strategic exercise of options: development cascades and overbuilding in real estate markets, Journal of Finance 51: 1653-1679. http:// dx.doi.org/10.1111/j.1540-6261.1996.tb05221.x

Grenadier, S. R. 2002. Option exercise games: an application to the equilibrium investment strategies of firms, Review of Financial Studies 15(3): 691-721. http://dx.doi.org/10.1093/rfs/15.3.691

Hamilton, B.; Schwab, R. 1985. Expected appreciation in urban housing markets, Journal of Urban Economics 18: 103-118. http://dx.doi.org/10.1016/00941190(85)90030-0

Hong, H.; Stein, J. C.; Yu, J. L. 2007. Simple forecasts and paradigm shifts, Journal of Finance 62: 1207-1242. http://dx.doi.org/10.1111/j.1540-6261.2007.01234.x

Kogan, L.; Ross, S. A.; Wang, J.; Westerfield, M. M. 2006. The price impact and survival of irrational traders, Journal of Finance 61(1): 195-229. http://dx.doi. org/10.1111/j.1540-6261.2006.00834.x

Kong, J. J.; Kwok, Y. K.; 2007. Real options in strategic investment games between two asymmetric firms, European Journal of Operational Research 181(2): 967-985. http://dx.doi.org/10.1016/j.ejor.2006.07.006

Lai, R. N.; Wang, K.; Zhou, Y. 2004. Sale before completion of development: pricing and strategy, Real Estate Economics 32(2): 329-357. http://dx.doi. org/10.1111/j.1080-8620.2004.00094.x

Lin, X. S. 2006. Introductory stochastic analysis for finance and insurance. Hoboken, NJ: John Wiley \& Sons. http://dx.doi.org/10.1002/0471793213

Mason, R.; Weeds, H. 2010. Investment, uncertainty and pre-emption, International Journal of Industrial Organization 28: 278-287. http://dx.doi.org/10.1016/j. ijindorg.2009.09.004

Meese, R.; Wallace. N. 1994. Testing the present value relation for housing prices: should I leave my house in San Francisco?, Journal of Urban Economics 35: 245-266. http://dx.doi.org/10.1006/juec.1994.1015
Mian, A.; Sufi, A. 2009. The consequences of mortgage credit expansion: evidence from the US mortgage default crisis, Quarterly Journal of Economics 124: 1449-1496. http://dx.doi.org/10.1162/ qjec.2009.124.4.1449

Nielsen, M. J. 2002. Competition and irreversible investment, International Journal of Industrial Organization 20: 731-743. http://dx.doi.org/10.1016/S01677187(01)00057-1

Ong, S. E.; Cheng, F. J.; Boon, B.; Sing, T. F. 2003. Oligopolistic bidding and pricing in real estate development: experimental evidence, Journal of Property Investment and Finance 21(2): 154-189. http://dx.doi. org/10.1108/14635780310469120

Pawlina, G.; Kort, P. M. 2006. Real options in an asymmetric duopoly: who benefits from your disadvantage, Journal of Economics and Management Strategy 15(1): 1-35. http://dx.doi.org/10.1111/j.15309134.2006.00090.x

Sargent, T. J.; Wallace, N. 1976. Rational expectations and the theory of economic policy, Journal of Monetary Economics 2: 169-183. http://dx.doi. org/10.1016/0304-3932(76)90032-5

Shaked, A.; Sutton, J. 1982. Relaxing price competition through product differentiation, Review of Economic Studies 49: 3-13. http://dx.doi.org/10.2307/2297136

Smit, H.; Trigeorgis, L. 2004. Strategic investment: real options and games. NJ: Princeton University Press.

Trigeorgis, L. 1996. Real options. Cambridge, MA: MIT Press.

Wang, K.; Zhou, Y. 2000. Overbuilding: a game-theoretic approach, Real Estate Economics 28(3): 493-522. http://dx.doi.org/10.1111/1540-6229.00810

Wang, K.; Zhou, Y. 2006. Equilibrium real options exercise strategies with multiple player: the case of real estate markets, Real Estate Economics 34(1): 1-49. http://dx.doi.org/10.1111/j.1540-6229.2006.00158.x

Williams, J. T. 1993. Equilibrium and options on real assets, Review of Financial Studies 6(4): 825-850. http://dx.doi.org/10.1093/rfs/6.4.825

Wong, K. P. 2007. The effect of uncertainty on investment timing in a real options model, Journal of Economic Dynamics \& Control 31: 2152-2167. http://dx.doi.org/10.1016/j.jedc.2006.07.002 